\title{
Pseudosarcomatous myofibroblastic proliferation of ureter
}

\author{
Seok Kwan Hong ${ }^{1 *}$, Chin Fong Wong ${ }^{2}$ and Yee Mun Lee ${ }^{1}$ \\ ${ }^{1}$ Urology Department, Tan Tock Seng Hospital, Singapore \\ ${ }^{2}$ Pathology Department, Tan Tock Seng Hospital, Singapore
}

\begin{abstract}
Pseudosarcomatous myofibroblastic proliferation is a rare lesion of the genitourinary tract. This rare entity has been reported in the bladder but an uncommon histology finding in the ureter. We report a rare case of pseudosarcomatous myofibroblastic proliferation of the ureter. It is a diagnostic challenge to differentiate it from a malignant ureter lesion. Surgical resection is the treatment of choice and the diagnosis is made on histology. There have been reports of recurrences in up to $10 \%$ of patients and a rare association with sarcomatoid urothelial carcinoma. Hence surgical resection of ureteric lesion and close follow up are main stay of management.
\end{abstract}

\section{Introduction}

Pseudosarcomatous myofibroblastic proliferation is a rare lesion that arises from submucosal stroma. It has been reported in the ureter, bladder, urethral and prostate [1]. It is also known as inflammatory myofibroblastic tumour (IMT), postoperative spindle cell nodule, inflammatory pseudotumour or pseudo sarcomatous fibromyxoid tumour. Pseudosarcomatous myofibroblastic proliferation of the ureter is uncommon and poses a diagnostic challenge as endoscopic examination and radiological imaging are not able to reliably differentiate between pseudosarcomatous myofibroblastic proliferation of ureter and a malignant ureteric tumour. The diagnosis is largely dependent on histologic findings

\section{Case Report}

A 63 year old Chinese male with horseshoe kidney, hypertension and hyperlipidaemia presented with 1 day history of acute left flank pain. At the time of consultation, no abnormality was demonstrated on clinical examination. Serum creatinine was mildly elevated at 132 umol/L but improved with hydration. CT Urogram showed a distal left ureteric stone and a $1 \mathrm{~cm}$ lobulated soft tissue lesion at the left mid ureter. There was no hydronephrosis. He subsequently passed out the stone. We proceeded to left ureteroscopy and found a non papillary soft tissue mass at the left mid ureter with calcification. Biopsy showed anucleated laminated keratin with dystrophic calcification. There was no clear evidence of malignancy. We repeated the left ureteroscopy in 6 weeks and repeated biopsy of that lesion. Histology revealed acute on chronic inflammation and squamous metaplasia. Urine cytology from the bladder and left ureter did not reveal any malignant cells.

The case was presented in the multi-disciplinary team meeting which suggested open segmental ureterectomy. Intraoperatively, we found a $1 \mathrm{~cm}$ soft tissue nodule at left mid ureter. Histology suggested a pseudosarcomatous myofibroblastic proliferation. We saw myxoid background with uniform spindle cells arranged in fascicle. No significant mitotic activity was seen. Immunohistologically it was positive for SMA but negative for desmin, ALK, S-100 protein and cytokeratin.

\section{Discussion}

Pseudosarcomatous myofibroblastic proliferation is a rare benign pathology of unknown cause which has been reported in the bladder, prostate, urethral and ureter. Most of the available medical literature describes pseudosarcomatous myofibroblastic proliferation in the bladder with very few reports about its presence in the ureter. Montgomery et al. found 1 case that involved the ureter with extension to the renal pelvis and had history of in dwelling ureteropelvic junction stent insertion prior to diagnosis [2]. Up to $25 \%$ of these lesions arise after antecedent trauma or surgical instrumentation but most lesions arise spontaneously with no history of trauma [3].

The majority of patients presented with haematuria and it usually involved the bladder. It is difficult to distinguish pseudosarcomatous myofibroblastic proliferation of ureter from a malignant ureteric tumour using endoscope or radiological imaging. Histopathology examination is the only reliable diagnostic tool to clinch the diagnosis. However, it is challenging to obtain adequate tissue sample from a ureteric lesion.

Our patient underwent two ureteroscopy and biopsy which yielded acellular keratin and acute on chronic inflammation with squamous metaplasia. Laser ablation failed to ablate the lesion completely. Our team was concerned about missing a malignancy. After discussion with patient and the multidisciplinary team, we proceeded with segmental ureterectomy. Intraoperatively, we found a $1 \mathrm{~cm}$ soft lobulated nodule at the left mid ureter. Histology showed a myofibroblast proliferation in a myxoid background. There was no mitotic activity seen. It was stained negative for anaplastic lymphoma kinase -1 (ALK) and desmin but positive for SMA. Staining for desmin and SMA can be variable in pseudosarcomatous myofibroblastic proliferation tumour. Pseudosarcomatous myofibroblastic proliferation has low metastatic potential but may recur in $10 \%$ to $25 \%$ of patients $[2,4]$. It can be locally aggressive. There is a report of concurrent sarcomatoid urothelial carcinoma but this is rare [2]. For these reasons, a surgical resection should be advocated with close follow up (Figures 1-5).

${ }^{\star}$ Correspondence to: Seok Kwan Hong, Urology Department, Tan Tock Seng Hospital, Singapore, E-mail: seok_kwan_hong@ttsh.com.sg

Received: May 22, 2018; Accepted: May 28, 2018; Published: May 31, 2018 


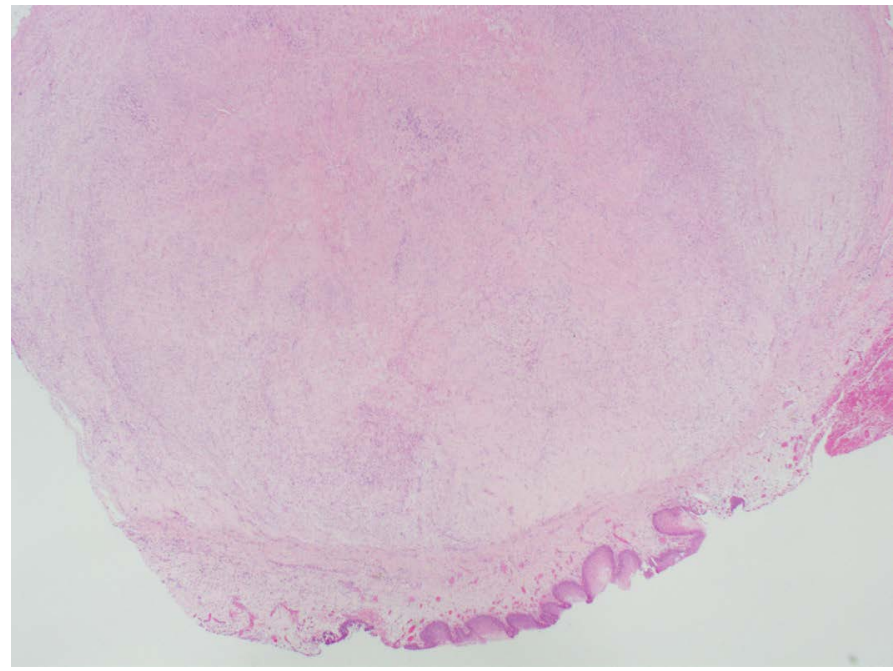

Figure 1. Mass seen under urothelium (H\&E, 20X)

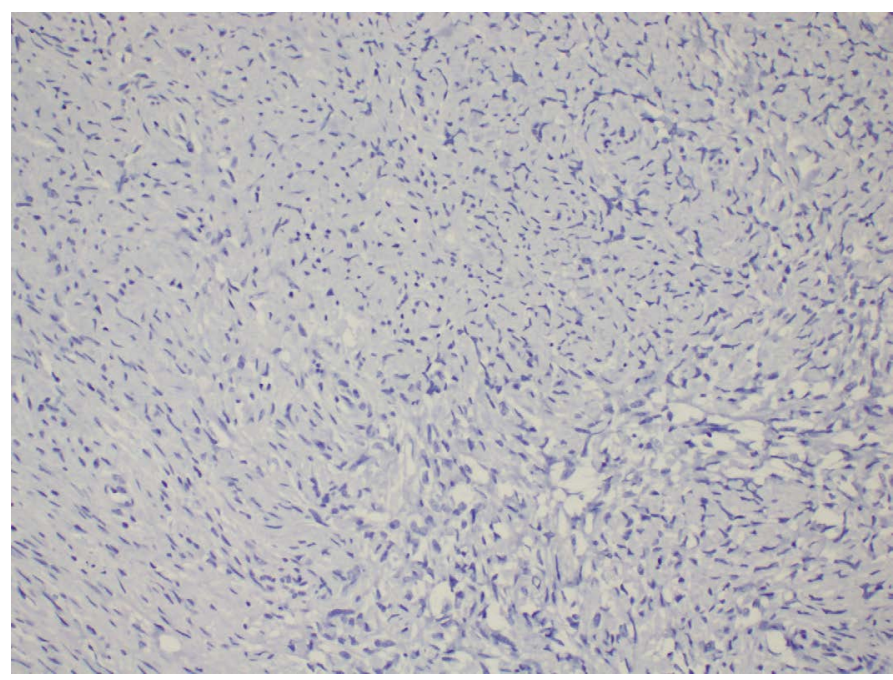

Figure 2. Immunohistochemistry shows negative staining with ALK (20X)

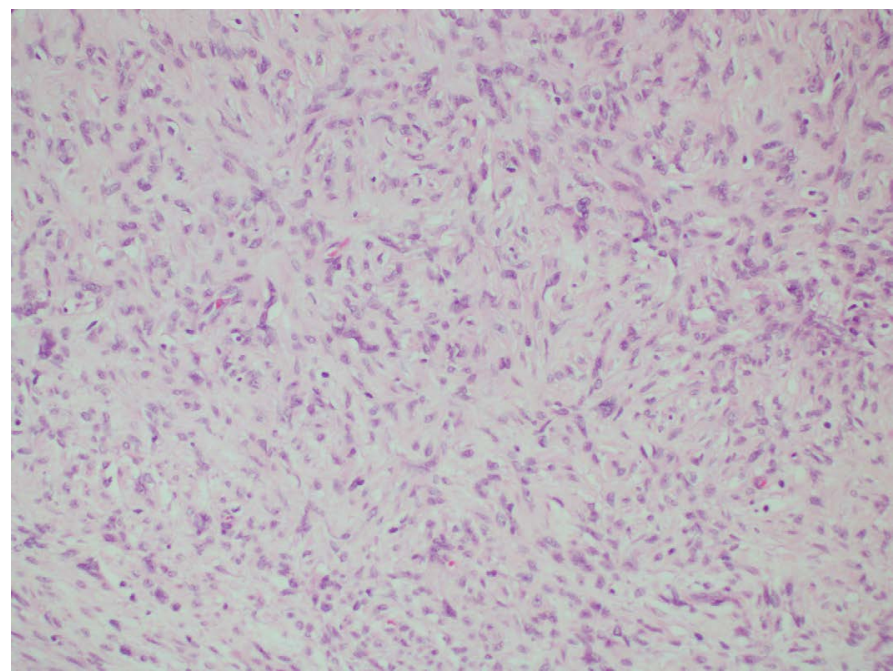

Figure 3. Myofibroblast proliferation (H\&E, 20X)

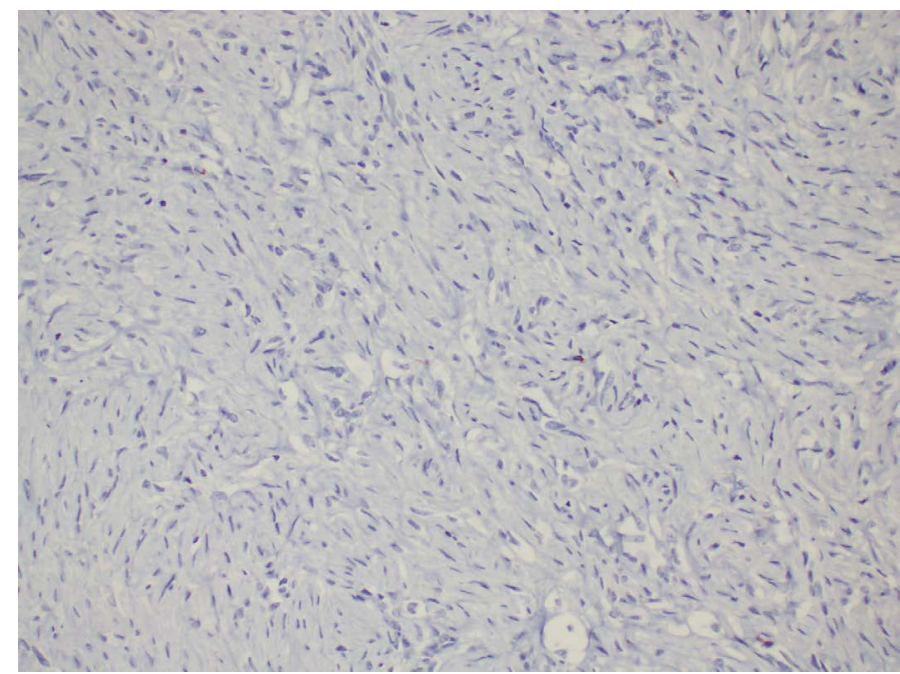

Figure 4. Immunohistochemistry shows negative staining with desmin (20X)

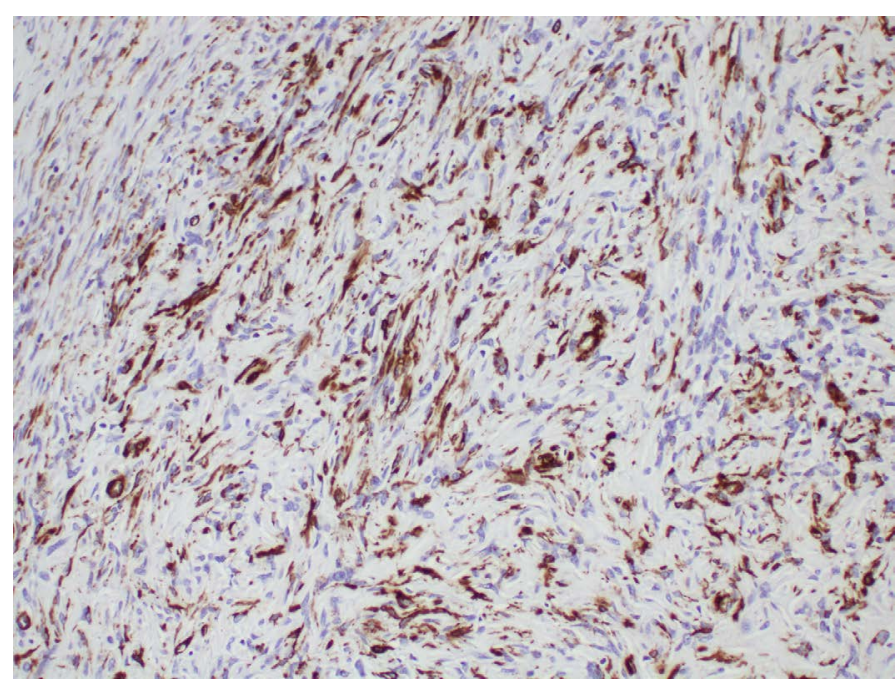

Figure 5. Immunohistochemistry shows positive staining with SMA (20X)

\section{Conclusion}

Pseudosarcomatous myofibroblastic proliferation of the ureter is a rare lesion of the genitourinary tract with few reported cases worldwide. It is challenging to differentiate it from a malignant ureter lesion with endoscopy or radiological imaging alone. There have been reports of recurrences in up to $10 \%$ of patients and a rare association with sarcomatoid urothelial carcinoma. Hence surgical resection of ureteric lesion and close follow up are main stay of management.

\section{Conflict of Interest}

The authors declare no conflicts of interest

\section{Acknowledgement}

No

\section{Financial Disclosure}

No 


\section{References}

1. Bostwick DG, Cheng L (2008) Urology Surgical Pathology. Elsevier Sciences 536.

2. Montgomery EA, Shuster DD, Burkart AL, Esteban JM, Sgrignoli A, et al. (2006) Inflammatory Myofibroblastic Tumors of the Urinary Tract: A Clinicopathologic Study of 46 Cases, Including a Malignant Example Inflammatory Fibrosarcoma and a Subset Associated With High-grade Urothelial Carcinoma. Am J Surg Pathol 30: 1502-12. [Crossref]
3. Goldblum JR (2006) Myofibroblastic proliferations of the genitourinary tract: Reactive pseudosarcomatous proliferations or inflammatory myofibroblastic tumors? Adv Anat Pathol 13.

4. In AUA website (Online). Pathology for Urologist Available: http://www.auanet.org education/auauniversity/education-products-and-resources/pathology-for-urologists/ urinary-bladder/mesenchymal-and-other-tumors/myofibroblastic-lesion Access date 01 May 2018

Copyright: (C2018 Hong SK. This is an open-access article distributed under the terms of the Creative Commons Attribution License, which permits unrestricted use, distribution, and reproduction in any medium, provided the original author and source are credited. 